\title{
Synthesis and Formation Mechanism of Textured MOF-5
}

\author{
Heather F. Greer, Yuehao Liu, Alex Greenaway, Paul A. Wright, and Wuzong Zhou* \\ EaStChem, School of Chemistry, University of St Andrews, St Andrews, KY16 9ST, United Kingdom.
}

\begin{abstract}
Textured and house-of-card structures develop on the surfaces of cubic MOF-5 crystals. The formation mechanism of these novel constructions are investigated based on characterisation of the intermediate specimens with different reaction time intervals using scanning and transmission electron microscopies. It is found that the growth of MOF-5 cubic crystals does not follow the classical route. MOF-5 nanocrystallites initially aggregate to form large polycrystalline cubes, followed by surface rearrangement of the nanocrystallites into a textured structure consisting of nanowires lying along the [100], [010], [110] and [1 10$]$ zone axes, if the face of the cube is defined to be (001). Further crystal growth leads to a house-of-cards structure constructed by interpenetrated nanoplates with their shortest axis parallel to [110] and [110] axes. Evidence of a reversed crystal growth process is also observed. MOF-5 cubes with a single crystal-like appearance are achieved with extended reaction times despite their hidden three tier structure containing varying porosities hidden underneath.
\end{abstract}

\section{INTRODUCTION}

Metal organic frameworks (MOFs) are a class of porous materials constructed from organic ligands coordinated to metal ions or metal nanoclusters to form one-, two- or threedimensional frameworks. ${ }^{1}$ Although MOFs are as porous as zeolites, the size, shape and adsorption performance of the pores are distinguishable. MOFs tend to be less thermodynamically stable than most inorganic microporous materials owing to the presence of organic linkers and hence are limited in conventional high-temperature catalyst applications. However their very high specific surface areas, tuneable porosity and chemically tuneable structures due to the diversity in metal and functional groups attracts attention in the fields of hydrogen and carbon dioxide storage, ${ }^{2,3}$ gas purification, ${ }^{4}$ biomedicine ${ }^{5}$ and sensing. ${ }^{6}$ Over the last two decades, intense research has been carried out on MOFs which has broadened and enriched the diversity of porous materials. ${ }^{7}$ More recently nanoand mesostructures of MOFs have become attractive due to their unique properties compared with their corresponding bulk material. $^{8,9}$

MOF-5, the first robust MOF to be prepared by Yaghi and co-workers, ${ }^{10}$ remains one of the most frequently synthesised and characterized MOFs with a composition of $\mathrm{Zn}_{4} \mathrm{O}(\mathrm{BDC})_{3}$, where $\mathrm{BDC}=1,4$-benzenedicarboxylate. It has a structure consisting of $\left[\mathrm{Zn}_{4} \mathrm{O}\right]^{6+}$ clusters in octahedral subunits joined by BDC linkers, to give an extended porous 3-dimensional framework. This special structure gives MOF-5 a large Brunauer-Emmett-Teller (BET) surface area of up to 3900 $\mathrm{m}^{2} \mathrm{~g}^{-1}$. ${ }^{11}$ Solvothermal method ${ }^{12,13}$ is most commonly used to prepare MOF-5 but a number of other methods including microwave, ${ }^{14}$ sonochemical ${ }^{15}$ and synthesis via substrates ${ }^{16}$ have also be used. The zinc precursor, i.e. nitrate, sulphate, acetate, oxide or chloride, for the synthesis of MOF-5 must be carefully selected as this is known to have a significant effect on the purity and crystallinity of the product. ${ }^{13}$ It has been noticed that even if the 'same' synthetic conditions are used, it is still not easy to achieve the same quality of MOF-5 products.

More and more researchers have put emphasis on adjusting the pore structure and pore surface of MOF-5 to refine its performance for various applications, especially for gas storage. ${ }^{17}$ In particular, MOF-5 has been extensively investigated for $\mathrm{H}_{2}$ storage applications in fuel cells. ${ }^{18}$ One strategy is to increase the complexity of the crystals by producing crystals with a hierarchical structure and a high crystal ordering. A typical way to achieve this is to add a foreign species, usually a mono- or bidentate ligand, such as a carboxylate anion. Yaghi and co-workers ${ }^{19}$ recently demonstrated that the addition of a modulator, 4-(dodecyloxy) benzoic acid (DBA) in carefully controlled amounts can alter the crystals of MOF-5 from a homogeneous single crystal to those with a hierarchical arrangement of meso- and macropores. The meso- and macropores either permeated the whole crystal to make a sponge-like crystal or enclosed a thick crystalline microporous shell to make pomegranate-like crystals. These MOF-5 crystals, attributing various tiers of porosities, were found to have robust architectures and, in the pomegranate-like crystal, a superior $\mathrm{CO}_{2}$ adsorption property to the original single crystals of MOF-5. While the strategy of employing controlled amounts of DBA showed that the porosity can be manipulated without losing the original crystallinity, the formation mechanism of these special MOF-5 crystals has not been fully understood.

Indeed, most reports on MOFs pay attention to the quality of the final products. Very few reported cases exemplifying the step-by-step crystal growth process of MOFs are known. The limited knowledge on the nucleation and early stage crystal growth of MOFs greatly restricts our capabilities of the crystal engineering of these materials. In our previous work, ${ }^{20}$ MOF-5 was re-produced using an established method, ${ }^{21}$ at a slightly lower temperature $\left(110^{\circ} \mathrm{C}\right.$ instead of $\left.120^{\circ} \mathrm{C}\right)$ to slow down the reaction rate. The synthetic system is simple, only containing $\mathrm{Zn}\left(\mathrm{NO}_{3}\right)_{2} \bullet 6 \mathrm{H}_{2} \mathrm{O}$ and 1,4-benzenedicarboxylic acid (1,4$\mathrm{BDC})$ as precursors dissolved in $\mathrm{N}, \mathrm{N}$-dimethylformamide (DMF). It was found that the earliest detected crystalline particles are $\mathrm{Zn}_{5}\left(\mathrm{NO}_{3}\right)_{2}(\mathrm{OH})_{8} \cdot 2 \mathrm{H}_{2} \mathrm{O}$ nanoplatelets. These nanoplatelets with surface-adsorbed 1,4-BDC molecules loosely aggregate into layered inorganic/organic composite particles. MOF-5 crystallites formed inside these composite particles through a phase transformation and further aggregated into large polycrystalline cubes. Surface re-crystallisation led to a single crystalline shell, followed by an extension of crystallisation towards the cores, demonstrating another example of 
the reversed crystal growth route, but the first to be discovered in MOFs. ${ }^{22}$ It is interesting to discover that when the MOF-5 cubes were left in the synthetic solution for $5 \mathrm{~h}$ at room temperature, a woven coating layer consisting of orientated MOF5 nanowires developed on the smooth surface, leaving its formation mechanism yet to be studied. ${ }^{20}$

In the present work, we tried to re-synthesise sponge-MOF-5 using the method developed by Yaghi and co-workers. ${ }^{19} \mathrm{We}$ found, at an intermediate growth stage, the cubes of MOF-5 have a textured surface structure consisting of orientated nanowires arranged along $<100>$ and $<110>$ directions, forming a 'Union Jack'-like pattern. The nanowires further grew inwards to form some interpenetrated nanoplates, showing a house-ofcards structure at the surface. At extended growth times the crystals underwent surface re-crystallisation to form a single crystal shell as the crucial stage of a reversed crystal growth process. The mechanism of such a novel morphology evolution is discussed.

\section{EXPERIMENTAL SECTION}

Sample preparation. All chemicals were purchased from commercial sources, and were used without further purification, these including $\mathrm{Zn}\left(\mathrm{NO}_{3}\right)_{2} \bullet 6 \mathrm{H}_{2} \mathrm{O}$ (98\%, Fisher Scientific), 1,4-benzenedicarboxylic acid (1,4-BDC) (99\%, Alfa Aesar), 4-(dodecyloxy)benzoic acid (DBA) (99\%, Sigma Aldrich), N,N-diethylformamide (DEF) (99\%, Acros Organics), dimethylformamide (DMF) (99\%, Acros Organics), tetrahydrofuran (THF) (reagent grade, Fisher Scientific).

The MOF-5 specimens were prepared according to the previously reported two-step solvothermal synthesis method consisting of an aging step followed by initiation at increased temperature. ${ }^{19} \mathrm{Zn}\left(\mathrm{NO}_{3}\right)_{2} \cdot 6 \mathrm{H}_{2} \mathrm{O}(336 \mathrm{mg}, 1.13 \mathrm{mmol})$ and $1,4-$ BDC (41.5 mg, $0.25 \mathrm{mmol})$ were dissolved in DEF $(5 \mathrm{~mL})$ in a $7 \mathrm{~mL}$ vial at room temperature. Secondly, DBA $(76.6 \mathrm{mg}$, $0.25 \mathrm{mmol}$ ) was added and the solution was stirred for $24 \mathrm{~h}$. The solution was clear without any precipitates. Following this, the vial was sealed and placed in an oil bath at $150{ }^{\circ} \mathrm{C}$ for 20 min to yield pale yellow crystals. After cooling, the precipitate was collected by centrifugation, washed several times with $\mathrm{DMF}$, and then dried in a vacuum oven at $60{ }^{\circ} \mathrm{C}$. In addition, samples were also synthesised with longer reaction times of 25 $\min , 30 \mathrm{~min}, 3 \mathrm{~h}, 6 \mathrm{~h}, 24 \mathrm{~h}, 40 \mathrm{~h}$ and $48 \mathrm{~h}$. To study the effect of DBA, a 30 min specimen was prepared in the absence of DBA.

Prior to characterisation by nitrogen sorption the MOF-5 product was immersed in THF for 2 days, during which time the solvent was decanted and replenished twice with fresh solvent.

Sample characterization. Powder X-ray diffraction (XRD) was performed on either a PANalytical Empyrean diffractometer, using $\mathrm{Cu} \mathrm{K \alpha}$ radiation $(\lambda=1.5418 \AA)$ for solvated samples, or a Stoe STAD I/P diffractometer using $\mathrm{Cu} \mathrm{Ka} 1 \mathrm{X}$ radiation (1.54056 $\AA$ ) for sealed capillaries containing desolvated sample. Analysis of the powder XRD patterns was carried out using Highscore plus software and GSAS suite of software. $^{23}$ Thermogravimetric analysis-mass spectrometry (TGMS) measurements were carried out on a Netzsch Jupiter STA 449C thermobalance, equipped with a Pfeiffer Vacuum ThermoStar mass spectrometer (MS) using a heating rate of $5{ }^{\circ} \mathrm{C}$ $\min ^{-1}$ under $\mathrm{O}_{2}$. An isotherm was carried out at $110^{\circ} \mathrm{C}$ for 0.5 $\mathrm{h}$ and at $200{ }^{\circ} \mathrm{C}$ for $1 \mathrm{~h}$. Examination of the porosity, the Brunauer-Emmett-Teller (BET) surface area and the pore vol- ume were measured by $\mathrm{N}_{2}$ adsorption/desorption isotherms at $196{ }^{\circ} \mathrm{C}$ on a Micromeritics Tristar II 3020 instrument. Scanning electron microscopic (SEM) images of the specimens were obtained using a JEOL JSM-6700F field-emission gun (FEG) microscope, operating at 1 to $5 \mathrm{kV}$ with gentle mode. To overcome beam charging problems, the specimen surface was coated with a thin gold film using a Quorum Technologies Q150R ES sputter coater/carbon coater prior to SEM analysis. The FEG-SEM is equipped with an Oxford INCA system for energy dispersive X-ray spectroscopy (EDX), which was applied for examination of the local chemical composition of the specimens. Transmission electron microscopic (TEM) images and selected area electron diffraction (SAED) patterns were attained using a JEOL JEM-2011 electron microscope fitted with $\mathrm{LaB}_{6}$ filament operating at an accelerating voltage of $200 \mathrm{kV}$. This electron microscope is also equipped with an Oxford Link ISIS SemiSTEM EDX system. The TEM and high-resolution TEM (HRTEM) images were recorded using a Gatan 794 CCD camera.

\section{RESULTS AND DISCUSSION}

Using the synthetic method outlined in the experimental section, large cubic MOF-5 crystals (20-70 $\mu \mathrm{m}$ in diameter) can be produced after a short reaction time, e.g. $30 \mathrm{~min}$ (Figure S1a in Supporting Information). A typical EDX spectrum (Figure S1b in Supporting Information) recorded from this sample displays a high concentration of zinc and contains significant peaks corresponding to carbon and oxygen. However, many wide cracks can be seen on the surfaces of the cubes, indicating that the cubes are not single crystals. Almost perfect cubes are found in the sample with a reaction time of $48 \mathrm{~h}$ (Figure 1a). Surprisingly, when the cubes are broken, a

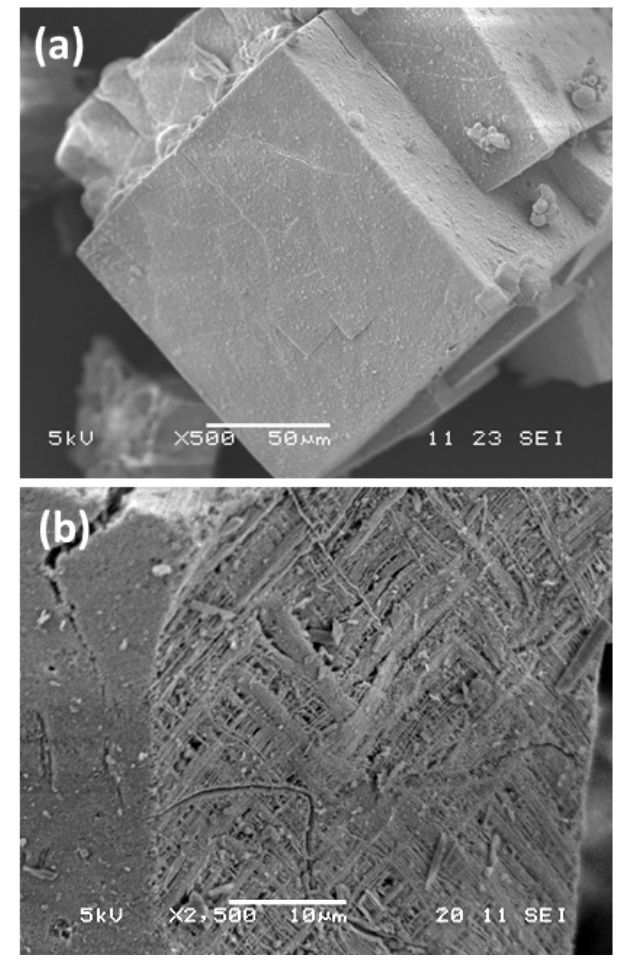

Figure 1 SEM images of sample with growth time of $48 \mathrm{~h}$, showing (a) MOF-5 cubes and (b) a broken cube revealing a core-shell structure, with a textured core and a single crystal shell. 
core-shell structure is revealed. Underneath a very thin single crystal shell, the core consists of orientated nanowires and nanoplates, forming a textured structure (Figure 1b). It is of great interest to find out how this novel structure developed during the crystal growth process.

According to the XRD patterns, all the specimens are monophasic and the patterns can be indexed approximately in the cubic unit cell expected for MOF-5 with the unit cell parameter of $\mathrm{a}=25.83 \AA$, space group $\mathrm{Fm} \overline{3} \mathrm{~m}$. More detailed investigation (see below) suggests the true symmetry to be rhombohedral. The intensity distribution of the peaks, and especially the relative intensity of the (200) cubic and (220) cubic reflections, is similar to that reported by Huang ${ }^{24}$ and reproduced by Hafizovic, ${ }^{21}$ for a form of MOF-5 in which disordered $\mathrm{Zn}(\mathrm{OH})_{2}$ was determined to be present in the pores of MOF-5. It is noted that an increase in crystallinity was observed in the XRD patterns of specimens with reaction times ranging from $20 \mathrm{~min}$ (Figure 2a) to $3 \mathrm{~h}$ (Figure 2c).

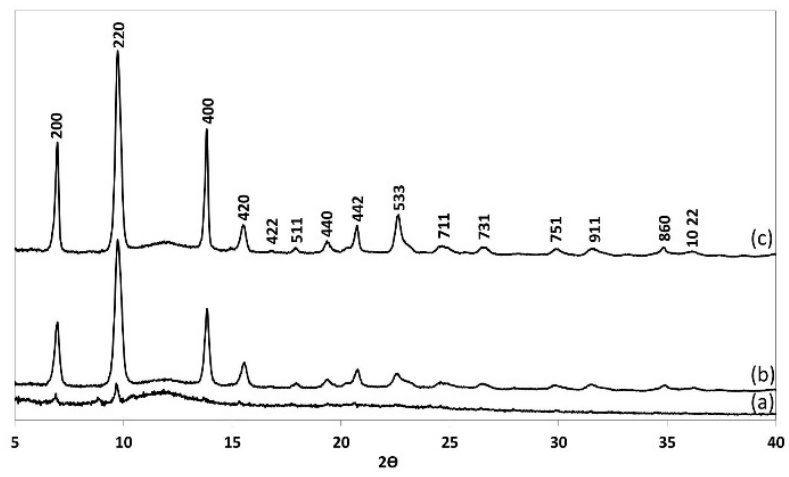

Figure 2 XRD patterns of MOF-5 specimens prepared with growth times of (a) $20 \mathrm{~min}$, (b) $30 \mathrm{~min}$, and (c) $3 \mathrm{~h}$. The peaks in pattern (c) have been indexed to cubic MOF-5.

In our previous synthesis, the precursors $\mathrm{Zn}\left(\mathrm{NO}_{3}\right)_{2} \cdot 6 \mathrm{H}_{2} \mathrm{O}$ and 1,4-benzenedicarboxylic acid (1,4-BDC) were dissolved in the solvent $\mathrm{N}, \mathrm{N}$-dimethylformamide (DMF) without adding any other chemicals. ${ }^{20} \mathrm{DMF}$ is a hygroscopic liquid and can deprotonate $\mathrm{H}_{2} \mathrm{O}$, enhancing the formation of $\mathrm{Zn}_{5}\left(\mathrm{NO}_{3}\right)_{2}(\mathrm{OH})_{8} \cdot 2 \mathrm{H}_{2} \mathrm{O}$ nanoplates. In the present work, on the other hand, N,N-diethylformamide (DEF) was used as solvent, which is a relatively weaker proton acceptor. More importantly, the addition of 4-(dodecyloxy)benzoic acid (DBA) greatly suppressed the formation of $\mathrm{OH}^{-}$anions. Therefore, the intermediate phase $\mathrm{Zn}_{5}\left(\mathrm{NO}_{3}\right)_{2}(\mathrm{OH})_{8} \cdot 2 \mathrm{H}_{2} \mathrm{O}$ was not observed.

The SEM and TEM images from the samples rationalized the change of crystallinity. In the specimen collected after a reaction time of $20 \mathrm{~min}$, nanoplates with a dimension in the range of $0.4-1 \mu \mathrm{m}$ appeared as shown in Figure 3a. The thickness of the plates is approximately $20 \mathrm{~nm}$ as measured from the plate marked by the white arrow in Figure 3b. Although the edges of nanoplatelets terminate with right angles, as indicated by the black arrows in Figure 3b, which is consistent with the cubic structure of MOF-5, no typical cubic morphology was observed. A possible explanation for the nanoplate formation is that the particle surface is coated with long chain organic molecules, such as DBA, as we observed in the early stage crystal growth of zeolite analcime. ${ }^{22} 1,4-\mathrm{BDC}$ molecules have two carboxylic groups $(\mathrm{COOH})$ attached at the opposite sites of benzene ring and can therefore act as a build- ing unit for MOF-5. DBA, on the other hand, has only one carboxylic group at one end of benzene with a long alkyl chain at the opposite end. Consequently, they adsorb on the crystal surface as a stopper, avoiding further crystal growth.

Bearing in mind that the ratio of $\mathrm{Zn}: \mathrm{BDC}$ in MOF-5 is $4: 3$, but the ratio of $\mathrm{Zn}$ : BDC : DBA added in the synthetic solution is $4.52: 1: 1$. That means $\mathrm{Zn}$ is in excess (and can explain the presence of zinc species suggested from the XRD intensities) and DBA likely participates alongside BDC during the combination with $\mathrm{Zn}$ oxide clusters. Most DBA molecules must stay on the crystal surface with the most stable attachment of DBA occurring on the $\{100\}$ surfaces of MOF-5. The DBA molecules can form a high density monolayer due to the inter-molecular interaction between their long alkyl chains, although bonding of DBA on other surfaces cannot be ruled out, e.g. on the $\{110\}$ surfaces, where the loose ends of two adjacent DBA molecules are close to each other to stabilise the attachment. Consequently, the formation of rectangular nanoplates with the exposed (100) surface as shown in Figure 3b can be understood. At $25 \mathrm{~min}$, when these nanoplates aggregated into some large particles, they tended to self-orientate, forming a cubic morphology containing a dense surface layer (Figure S2a,b in Supporting Information). Figure 3c shows self-orientated nanoplates on one face of a cubic particle. This self-orientation can be regarded as the driving force for the formation of the polycrystalline cubes.
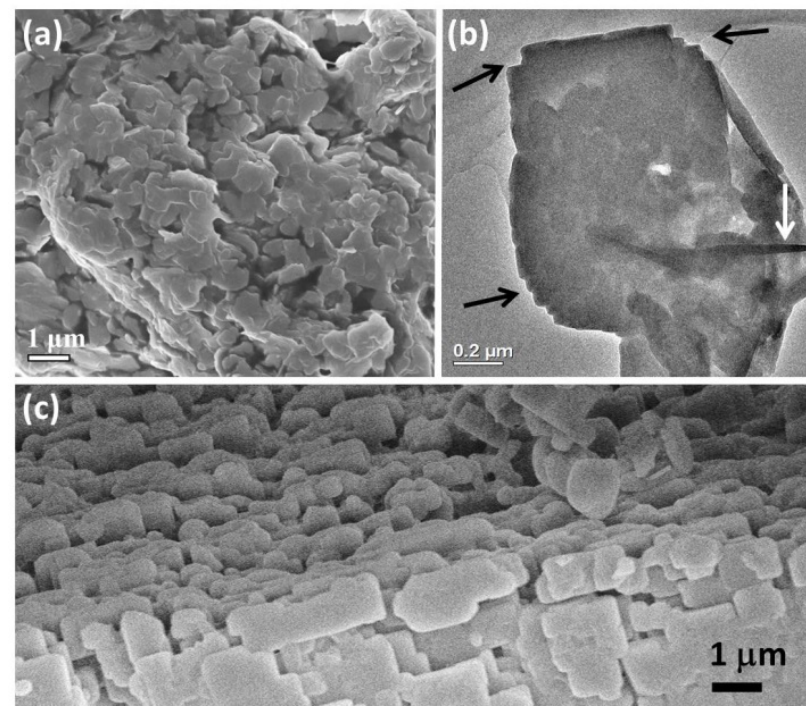

Figure 3 (a) SEM and (b) TEM images of MOF-5 nanoplates in 20 min sample. (c) SEM image of an edge of a polycrystalline cube in the 25 min specimen, showing self-orientation of the nanoplates on the (100) surface.

Increasing the reaction time allowed a morphology evolution from nanoplate to small cubic and spherical particles, $200 \mathrm{~nm}$ to $1 \mu \mathrm{m}$ in diameter, as shown in Supporting Information, Figures S2b and S2c. Further crystal growth did not lead to a single crystal shell. Instead, particles on the surface lined up along [100], [110] and symmetry-equivalent directions, forming a textured structure with a 'Union Jack'-like pattern (Figure 4). The enlarged SEM image shows a multi-layer textured structure, forming macropores. The discontinuity observed in the morphology of the nanowires, especially those along the $<110>$ directions as shown in Figure $\mathbf{4 b}$ confirms that these 
nanowires were formed via a linear connection of the particles, rather than growing from single seeds. More SEM images of these textured cubes are shown in Supporting Information, Figure S3. It is interesting to see that some cubes have missing corners and instead appear to have $\{111\}$ facets, on which a microrod array covers the whole face (Figure S3c in Supporting Information).
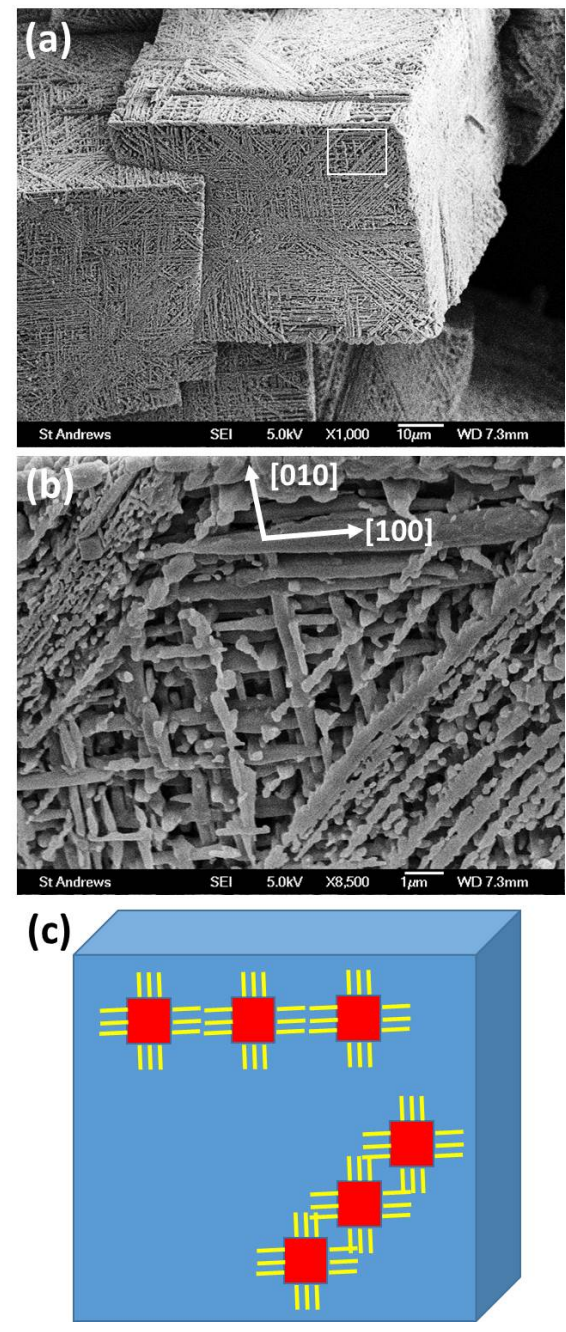

Figure 4 (a) SEM image of a typical textured MOF-5 cube. (b) A higher magnification SEM image of the marked area in (a) showing the textured surface in more detail. (c) Schematic drawing of two different connection manners of the MOF-5 nanocubes (red squares) with surface ligand molecules (yellow lines).

A close inspection of the (001) surface in Figure $\mathbf{4 b}$ allows us to find that most of the nanowires are straight and do not interpenetrate each other, but align on the surface with alternative orientations. The nanowires along the [100] and [010] directions have a smooth surface and constant diameter, while most of those along the [110] and symmetry-equivalent zone axes have a nodular morphology. We assume all the nanowires are made from a one dimensional connection of nanocubes containing a layer of DBA molecules on their surface, and the orientation of the nanocubes matches the crystallographic orientation of the [001] surface. The nanowires along the [100] and [010] directions form by a face-to-face connection and therefore appear to have a higher crystallinity. The others are constructed via an edge-to-edge connection. The two different morphologies of the nanowires can be understood as elucidated in Figure 4c. The almost perfect consistency of the orientations of the nanowires indicates again that the nanocubes in the aggregated particles are self-orientated. It is worth noting that the inter-axis angle between the [100] and [010] is $90^{\circ}$. The appearance of a larger angle in the SEM image of Figure 4b is because the surface is tilted. This is also the case in Figures $5 b$ and $5 c$.

A cubic morphology would be typical for MOF-5 which has a cubic structure according to the Bravais-Friedel-DonnayHarker (BFDH) law, ${ }^{25-27}$ and the Hartman-Perdok theory, ${ }^{28}$ in the case of the growth of free single crystals (kinetically controlled process); or according to the Curie-Wulff theorem, ${ }^{29,30}$ where a minimised surface free energy is considered (thermodynamically controlled). However, the formation of a cubic shape with a polycrystalline porous surface (see Figure 4a as an example) cannot be explained by any of these established theories. We believe the Curie-Wulff theorem is more general. However, in the case of polycrystalline polyhedral particles, we have to assume that the calculation of the surface free energy must include all molecules adsorbed on the surface in the real synthetic environment.
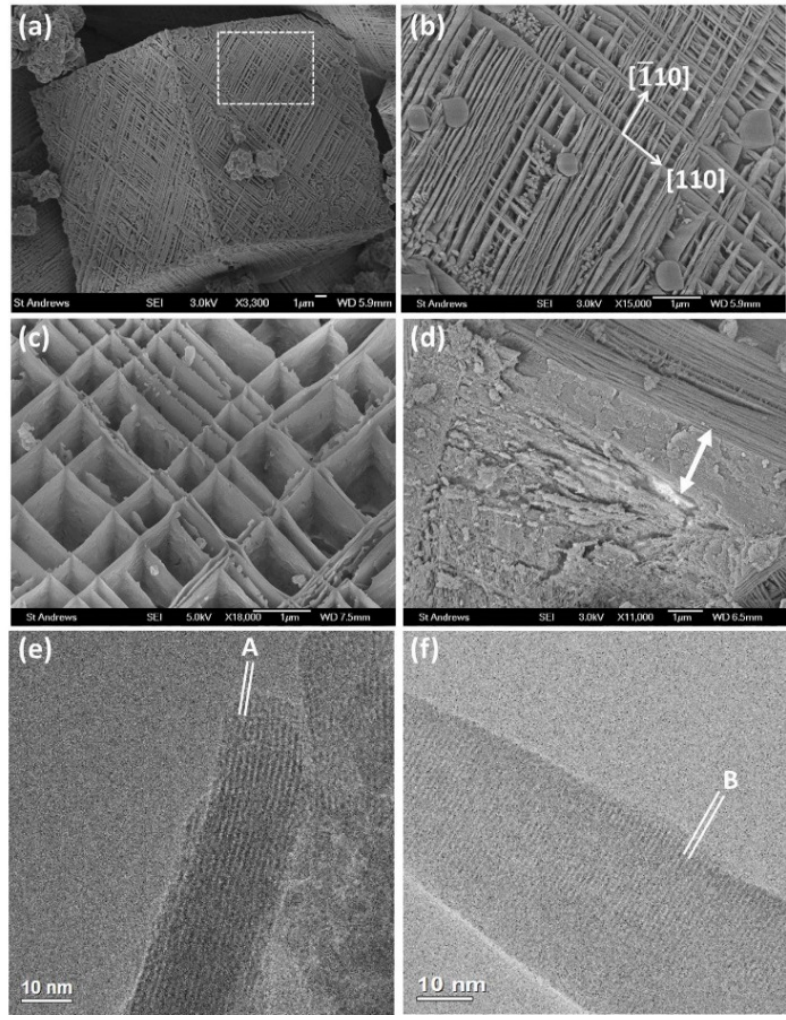

Figure 5 (a) SEM image of a typical MOF-5 cube from $30 \mathrm{~min}$ sample showing a woven surface structure. (b) Enlarged image of the marked area in (a). (c) A house-of-cards structure on a MOF-5 cube. (d) SEM image of a profile view of the nanoplates, visible when a cubic particle is fractured. (e) and (f) HRTEM images of fragments of the nanoplates. The measured d-spacings are A, 12.85 and B, $9.70 \AA$ Á.

With further increase of the reaction time to $30 \mathrm{~min}$, the nanowires grew inwards to form nanoplates, normal to the $\{100\}$ surface of the cubes. Figure 5a shows a typical cube of 
MOF-5 with such a surface structure. Figures 5b and 5c are the corresponding high magnification SEM images. It is noticed that all the nanoplates lie down along the [110] and [1 10$]$ directions. No nanoplates are present along the $\{100\}$ directions. The nanoplates interpenetrate each other at $90^{\circ}$, forming a house-of-cards surface structure. From these images, the thickness of the nanoplates is typically about $25 \mathrm{~nm}$ but can be as thick as $50 \mathrm{~nm}$, while the width is about $2 \mu \mathrm{m}$ (Figure 5d).

The cubes are still polycrystalline, consisting of nanocrystallites in the core (Figure S4 in Supporting Information).

TEM is a valuable characterisation technique for MOFs but their high organic content makes them sensitive to the electron beam and therefore they decompose under electron beam irradiation within several seconds. This usually makes it unfeasible to obtain HRTEM images unless some special conditions are used. A number of methods such as dehydrating, annealing with an electron beam and application of a low-dose electron beam have been outlined by Greer and Zhou. ${ }^{31}$ By using these techniques it is possible to keep some beam sensitive crystal structures intact under the electron beam irradiation for long enough to obtain HRTEM images. For example, reasonably good HRTEM images have been obtained from zeolites, $\mathrm{C}_{60} /$ trimethylbenzene composite, and MOFs. ${ }^{32-35}$ Using a very low dose of electron beam, some HRTEM images of fragments of MOF-5 nanoplates were obtained as shown in Figures 5e and 5f. Although some structural degradation is visible in the HRTEM images, weak crystalline fringes are observed along the profile and long axis of the nanoplates. The fringes with a d-spacing of $12.85 \AA$ in Figure $5 \mathbf{e}$ can be indexed to the (200) planes, while those in Figure 5f with a dspacing of $9.70 \AA$ can be indexed to the (220) crystal planes of cubic MOF-5. Therefore, the faces of the nanoplates can be identified to be the $\{220\}$ planes. In other words, the orientation of the nanoplates on the cube surface matches perfectly with the geometry of the cubes.

According to the Curie-Wulff theorem, re-crystallisation of disordered particles should form a single crystal shell to achieve a minimum surface energy. Indeed, we have observed such a phenomenon in many systems. For example, in zeolite analcime, an icositetrahedral single-crystal shell with $24\{211\}$ facets forms, ${ }^{22}$ in zeolite A a cubic shell develops, ${ }^{36,37} \mathrm{ZnO}$ forms a hexagonal prism shell, ${ }^{38}$ and calcite forms a rhombohedral shell, ${ }^{39}$ etc. In several of these systems, extended thermal treatment generated real single crystals through an extension of the crystallisation from the surface to the core, following the so-called reversed crystal growth route. However, in the present work, generation of a highly porous surface with a textured and house-of-cards structures made surface recrystallisation much more difficult. A possible reason is the low density and upright orientation of the nanoplates in the textured surface layer. A coating of DBA molecules on both surfaces of the MOF-5 plates would stablise the nanoplates and make it more difficult for the plates to lie down on the surface of the particle.

As Yaghi and co-workers reported, ${ }^{19}$ 4-(dodecyloxy)benzoic acid has dual functions, the carboxyl functional group to bind to the metal which is the most important chemical bonding in the MOF-5 structure and secondly, the alkyl chain for space filling. On the other hand, the simpler synthetic system of $\mathrm{Zn}\left(\mathrm{NO}_{3}\right)_{2} \cdot 6 \mathrm{H}_{2} \mathrm{O}$ and 1,4-BDC dissolved in DMF without adding DBA did not produce any house-of-card structures consisting of MOF-5 nanoplates. ${ }^{20}$ In the present work, we used DEF as the solvent instead of DMF and no DBA was added. The textured layer as shown in Figures 4 and 5 did not form. However, the MOF-5 cubes did consist of a large number of less rigid nanoplates as shown in Figure S5 in Supporting Information. The thickness of the nanoplates are similar to those in Figure 5c although their width is much shorter. The cubes prepared without DBA were found to have a much smaller crystallite size compared to MOF-5 cubes prepared in the presence of DBA (Figure S5c in Supporting Information). The reason is that the nanocrystallites were developed in some large disordered aggregates rather than in the solution as free crystals. DBA helps aggregation at early stages and the relatively higher concentrations of the precursor molecules/ions in the aggregates would increase the crystal growth rate. It is therefore indicated that DBA molecules may act as a promotor of aggregation. It also acts as surface ligand to block the extension of MOF- 5 crystals and act as a spacer in between the nanoplates.

To confirm the involvement of DBA, TGA-MS was performed (Figure S6 in Supporting Information). The first observed weight loss of $4.8 \%$ in the temperature range of 150 $200{ }^{\circ} \mathrm{C}$ is due to the removal of $\mathrm{H}_{2} \mathrm{O}$. A second weight loss of $3.8 \%$ during the isotherm period at $200{ }^{\circ} \mathrm{C}$ corresponds to the removal of surface adsorbed DBA molecules, confirmed by mass peaks of 115 and $135 \mathrm{~m} / \mathrm{z}$ corresponding to fragments of the alkyl chain in DBA. The final weight loss of $48.4 \%$ between $400-490{ }^{\circ} \mathrm{C}$ matches to the structural decomposition of MOF-5, leading to the final composition of $\mathrm{ZnO}$.

To determine the microporosity of the product MOF-5, nitrogen adsorption was carried out (Figure S7 in Supporting Information). The isotherms appear to have Type 1 character with a H3 hysteresis loop, typically stemming from the mesopores. A BET surface area of $787.2 \mathrm{~m}^{2} \mathrm{~g}^{-1}$ was measured from the 30 min sample by $\mathrm{N}_{2}$ sorption, which is much lower than that expected and observed for 'simple' MOF-5, where all pores are empty. The observed value is much closer to that reported by Huang ${ }^{24}$ for nanocrystalline MOF-5 and reproduced by Hafizovic ${ }^{21}$ in larger crystals which were amenable to single crystal X-ray diffraction studies. They identified the presence of $\mathrm{Zn}(\mathrm{OH})_{2}$ species in the pores that remain upon activation, significantly reducing the pore space, and also a much smaller amount of interpenetration. Catenation, an intergrowth of two or more frameworks is commonly found in highly-symmetric topologies and those prepared using long linkers. ${ }^{40}$

To examine this further, the MOF-5 prepared after 30 mins was heated at $130{ }^{\circ} \mathrm{C}$ under vacuum to remove residual solvent and its X-ray powder diffraction pattern collected. The pattern was better fitted by Le Bail analysis to a rhombohedral cell ( $a$ $=18.2487(6) \AA, c=44.674(2) \AA$ ) than to a cubic cell (Figure S8 in Supporting Information) and is close to that reported by Hafizovic ${ }^{21}$ for a 'low surface area MOF-5'. Furthermore, the PXRD intensity distribution was similar to that simulated for a sample in which Zn-bearing species occupied the pores with $50 \%$ occupancy, strongly reducing the porosity.

The presence of some interpenetration, which was also observed previously in MOF-5 samples prepared under similar conditions, is also likely. Interpenetrated MOF-5 frameworks are known to have better stability compared to noninterpenetrated MOF-5 frameworks. For example, Kim et al. ${ }^{41}$ demonstrated non-interpenetrated MOF-5 starts to decompose at $400{ }^{\circ} \mathrm{C}$, whereas the interpenetrated framework begins to decompose above $450{ }^{\circ} \mathrm{C}$. 
The house-of-cards texture of the surfaces of this MOF-5 material is of interest. Literature shows hierarchical interpenetrated arrangements of 2D nanoplates have been observed in zeolites with an MFI type framework. ${ }^{42-44}$ Zeolites with a socalled house-of-cards morphology where lamellae is arranged perpendicular to each other to define pores have successfully been prepared in the presence of complex organic quaternary ammonium salts and more recently using a low-cost green method via the introduction of N-methyl-2-pyrrolidone into the template-free synthesis. ${ }^{45}$ These hierarchical zeolites are reported to be particularly attractive both for fundamental research and their industrial applications since they possess three tiers of porosity: the intrinsic microporosity of the zeolite framework in unison with the mesoporosity within the plates and the macroporosity originating from the complex intergrown structure. $^{44}$

Nevertheless, the textured and house-of-cards structures in the present work are not thermodynamically stable in the mother liquor. When the reaction time was further increased, the space in between the nanoplates was filled by nanocrystallites (Figure 6a) and a new single crystal $\{100\}$ surface gradually formed on top of the house-of-cards structure. Figure $\mathbf{6 b}$ shows such a surface with an incomplete single crystal shell, making some nanoplates underneath visible, indicated by the arrows.
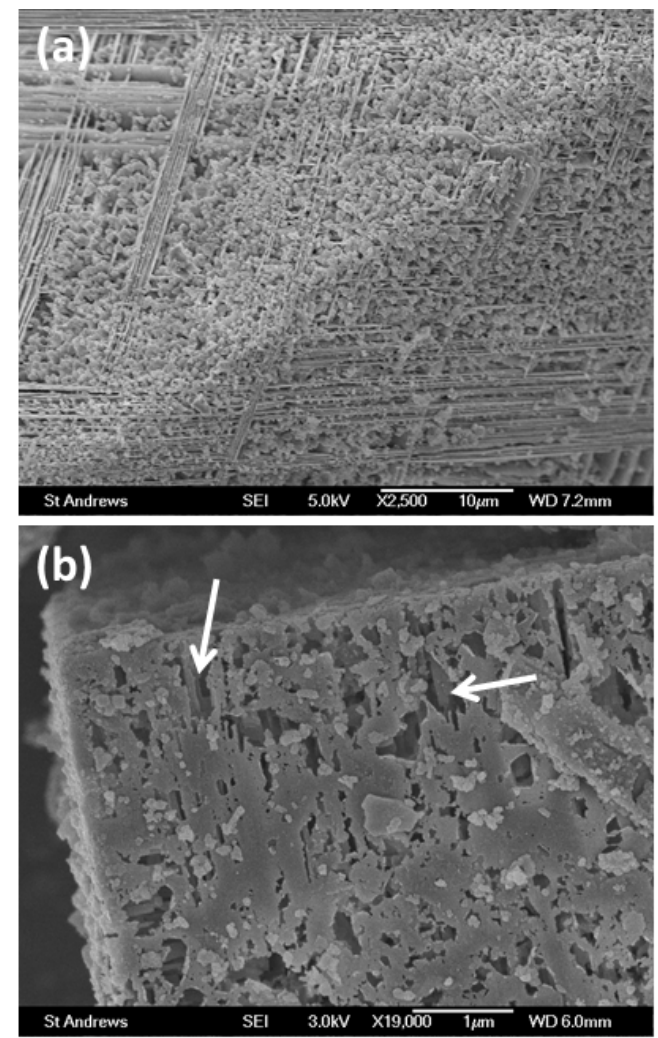

Figure 6 (a) SEM image of a MOF-5 cube from the $3 \mathrm{~h}$ sample. (b) SEM image of a MOF-5 cube from the $40 \mathrm{~h}$ sample, showing partial development of a single crystal shell. The arrows indicate nanoplates underneath the incomplete shell.

A complete single crystal cubic shell eventually formed with a longer growth time, e.g. $48 \mathrm{~h}$, as shown in Figure 1. It is expected that surface re-crystallisation would extend from the shell to the core via an Ostwald ripening process, performing again reversed crystal growth. ${ }^{22,46}$ However, this is beyond the scope of the present work.

In summary, a time dependent microstructural characterisation was carried out on MOF-5 specimens prepared when 4(dodecyloxy)benzoic acid was added as a modulator to a commonly used thermal method of $\mathrm{Zn}\left(\mathrm{NO}_{3}\right)_{2} \cdot 6 \mathrm{H}_{2} \mathrm{O}$, 1,4benzenedicarboxylic acid and DEF. The MOF-5 microcubes with textured and house-of-cards surface constructions have been fabricated as the intermediate phases towards singe crystalline MOF-5 cubes. Based on the experimental results obtained in the present work we are now able to propose a formation mechanism of these novel morphologies as elucidated in Figure 7.

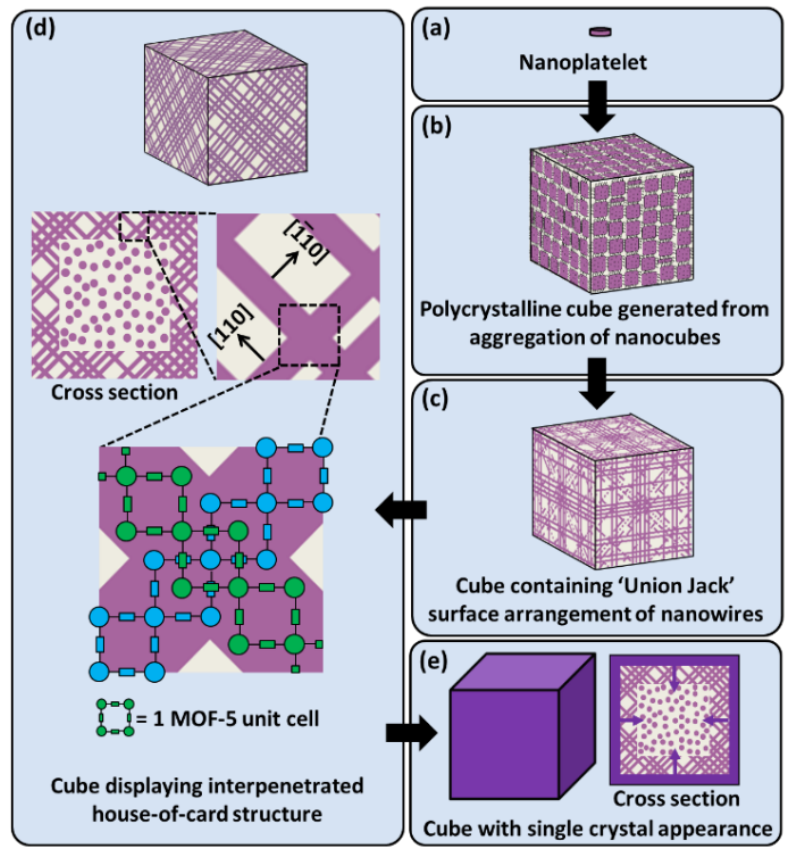

Figure 7. Proposed growth mechanism for cubes of MOF-5 displaying textured and house-of-cards exteriors.

The earliest detected crystalline phase was MOF-5 nanoplatelets (50-150 nm in dimension) (Figure 7a). Adsorption of DBA molecules to the (100) surface of MOF-5 plays an important role in the formation of these nanoplatelets. These nanoplatelets underwent rapid aggregation during heat treatment for $25 \mathrm{~min}$ to produce large polycrystalline microcubes (Figure 7b). The self-orientation of the nanoplatelets corresponds to the appearance of the cubic morphology. The nanoplatelets changed their morphology to nanocubes and underwent a one dimensional connection to form nanowires, lying down along the $<100>$ and $<110>$ directions, forming a 'Union Jack' type pattern on the $\{100\}$ surfaces (Figure 7c). DBA molecules again helped such a connection. The nanowires along the $<110>$ directions in the highly porous textured surface further grew inwards to form nanoplates. The nanoplates with a perpendicular relation interpenetrated each other, leading to a house-of-cards structure (Figure 7d). Although the nanoplates can penetrate towards the core to a depth of a few micrometers, the cores of the microcubes are still an arrangement of polycrystalline particles with random orientations. Surface re-crystallisation then took place to form a sin- 
gle crystalline shell, which is a crucial step of a reversed crystal growth process towards final single crystal microcubes of MOF-5 (Figure 7e). Consequently, if the reaction conditions are well controlled, textured and house-of-cards MOF-5 cubes can be fabricated. The latter has its majority surface terminated with the $\{220\}$ planes. These novel hierarchical structured MOF materials exhibiting a tiered structure of varying porosities may find their high potential in various catalytic applications, as was previously the case with hierarchical zeolites. ${ }^{43,45}$ MOF-5 structures combining intercrystalline mesopores with interpenetration has been shown to display significantly enhanced thermal stability and hydrogen storage capacity at 77 $\mathrm{K}$ and low pressure. ${ }^{47}$

\section{SUPPORTING INFORMATION}

More SEM images, EDX spectrum, TGA-MS plot, XRD pattern, $\mathrm{N}_{2}$ adsorption/desorption are supplied as Supporting Information. This material is available free of charge via the Internet at http://pubs.acs.org.

\section{AUTHOR INFORMATION \\ Corresponding Author \\ E-mail:wzhou@st-andrews.ac.uk}

\section{ACKNOWLEDGEMENTS}

HFG and WZ would like to thank the EPSRC for financial support on FEG-SEM equipment (EP/F019580/1) and a Platform (EP/K015540/1). YHL thanks Mr Ross Blackley for his assistance on using the SEM and TEM. The authors also thank Mrs Sylvia Williamson for performing TGA-MS and $\mathrm{N}_{2}$ sorption experiments.

\section{REFERENCES}

(1) Yaghi, O. M.; Li, H.; Davis, C.; Richardson, D.; Groy, T. L. Synthetic Strategies, Structure Patterns, and Emerging Properties in the Chemistry of Modular Porous Solids. Acc. Chem. Res. 1998, 31, 474-484.

(2) Suh, M. P.; Park, H. J.; Prasad T. K.; Lim, D. W. Hydrogen Storage in Metal-Organic Frameworks. Chem. Rev. 2012, 112, 782 835 .

(3) Zhang, D.-S.; Chang, Z.; Li, Y.-F.; Jiang, Z.-Y.; Xuan, Z.-H.; Zhang, Y.-H.; Li, J.-R.; Chen, Q.; Hu, T.-L.; Bu, X.-H. Fluorous Metal-Organic Frameworks with Enhanced Stability and High $\mathrm{H}_{2} / \mathrm{CO}_{2}$ Storage Capacities. Sci. Rep. 2013, 3, 3312.

(4) Li, J.-R.; Kuppler, R. J.; Zhou, H.-C. Selective Gas Adsorption and Separation in Metal-Organic Frameworks. Chem. Soc. Rev. 2009, $38,1477-1504$

(5) Horcajada, P.; Gref, R.; Baati, T.; Allan, P. K.; Maurin, G.; Couvreur, P.; Férey, G.; Morris, R. E.; Serre, C. Metal-Organic Frameworks in Biomedicine. Chem. Rev. 2012, 112, 1232-1268.

(6) Kreno, L. E.; Leong, K.; Farha, O. K.; Allendorf, M.; van Duyne, R. P.; Hupp, J. T. Metal-Organic Framework Materials as Chemical Sensors. Chem. Rev. 2012, 112, 1105-1125.

(7) Zhou, H. C.; Long, J. R.; Yaghi, O. M. Introduction to Metal-Organic Frameworks. Chem. Rev. 2012, 8, 673-674.

(8) Xin, Z.; Bai, J.; Pan, Y.; Zaworotko, M. J. Synthesis and Enhanced $\mathrm{H}_{2}$ Adsorption Prorperties of a Mesoporous Nanocrystal of MOF-5: Controlling Nano-/Mesostructures of MOFs to Improve their $\mathrm{H}_{2}$ Heat of Adsorption. Chem. Eur. J. 2010, 16, 13049 - 13052.

(9) Du, H.; Bai, J.; Zuo, C.; Xin, Z.; Hu, J. A Hierarchical Suprananostructure of HKUST-1 Featuring Enhanced $\mathrm{H}_{2}$ Adsorption Enthalpy and Higher Mesoporosity. CrystEngComm 2011, 13, $3314-3316$.
(10) Li, H.; Eddaoudi, M.; O’Keeffe, M.; Yaghi, O. M. Design and Synthesis of an Exceptionally Stable and Highly Porous Metal-Organic Framework. Nature 1999, 402, 276-279.

(11) Tranchemontagne, D. J.; Hunt, J. R.; Yaghi, O. M. Room Temperature Synthesis of Metal-Organic Frameworks: MOF-5, MOF-74, MOF-177, MOF-199, and IRMOF-0. Tetrahedron 2008, 64, 8553-8557.

(12) Chen, B.; Wang, X.; Zhang, Q.; Xi, X.; Cai, J.; Qi, H.; Shi, S.; Wang, J.; Yuan, D.; Fang, M. Synthesis and Characterization of the Interpenetrated MOF-5. J. Mater. Chem. 2010, 20, 3758-3767.

(13) Biemmi, E.; Christian, S.; Stock, N.; Bein, T. High-Throughput Screening of Synthesis Parameters in the Formation of the MetalOrganic Frameworks MOF-5 and HKUST-1. Micro. Meso. Mater. 2009, 117, 111-117.

(14) Choi, J. Y.; Kim, J.; Jhung, S. H.; Kim, H.-K.; Chang, J.-S.; Chae, H. K. Microwave Synthesis of a Porous Metal-Organic Framework, Zinc Terephthalate MOF-5. Bull. Korean Chem. Soc. 2006, 27, 1523-1524.

(15) Son, W.-J.; Kim, J.; Kim, J.; Ahn, W.-S. Sonochemical Synthesis of MOF-5. Chem. Commun. 2008, 6336-6338.

(16) Buso, D.; Nairn, K. M.; Gimona, M.; Hill, A. J.; Falcaro, P. Fast Synthesis of MOF-5 Microcrystals Using Sol-Gel $\mathrm{SiO}_{2}$ Nanoparticles. Chem. Mater. 2011, 23, 929-934.

(17) $\mathrm{Hu}, \mathrm{Y}$. H.; Zhang, l. Hydrogen Storage in Metal-Organic Frameworks. Adv. Mater. 2010, 22, E117-E130.

(18) Purewal, J.; Liu, D.; Sudik, A.; Veenstra, M.; Yang, J.; Maurer, S.; Müller, U.; Siegel, D. J. Improved Hydrogen Storage and Thermal Conductivity in High-Density MOF-5 Composites. J. Phys. Chem. C 2012, 116, 20199-20212.

(19) Choi, K. M.; Jeon, H. J.; Kang, J. K.; Yaghi, O. M. Heterogeneity within Order in Crystals of a Porous Metal-Organic Framework. J. Am. Chem. Soc. 2011, 133, 11920-11923.

(20) Zheng, C. M.; Greer, H. F.; Chiang, C.-Y.; Zhou, W. Z. Microstructural Study of the Formation Mechanism of Metal-Organic Framework MOF-5. CrystEngComm 2014, 16, 1064-1070.

(21) Hafizovic, J.; Bjørgen, M.; Olsbye, U.; Dietzel, P. D.; Bordiga, S.; Prestipino, C.; Lamberti, C.; Lillerud, K. P. The Inconsistency in Adsorption Properties and Powder XRD Data of MOF-5 Is Rationalized by Framework Interpenetration and the Presence of Organic and Inorganic Species in the Nanocavities. J. Am. Chem. Soc. 2007, 129, 3612-3620.

(22) Chen, X. Y.; Qiao, M. H.; Xie, S. H.; Fan, K. N.; Zhou, W. Z.; He, H. Y. Self-Construction of Core-Shell and Hollow Zeolite Analcime Icositetrahedra: A Reversed Crystal Growth Process via Oriented Aggregation of Nanocrystallites and Recrystallization from Surface to Core. J. Am. Chem. Soc. 2007, 129, 13305-13312.

(23) Larson, A. C.; Von Dreele, R. B. General Structure Analysis System (GSAS); Los Alamos National Laboratory: Los Alamos, 1994.

(24) Huang, L.; Wang, H.; Chen, J.; Wang, Z.; Sun, J.; Zhao, D.; Yan, Y. Synthesis, Morphology Control, and Properties of Porous Metal-Organic Coordination Polymers. Micro. Meso. Mater. 2003, 58, 105-114.

(25) Bravais, A. Études crystallographic. Gauthier-Villars, Paris, 1866.

(26) Friedel, M. G. Études Sur la Loi de Bravais. Bull. Soc. Fr. Mineral. Cristallogr. 1907, 30, 326-455.

(27) Donnay, J. D. H.; Harker D. A New Law of Crystal Morphology Extending the Law of Bravais. Am. Mineral. 1937, 22, 446-467.

(28) Hartman, P.; Perdok, W. G. On the Relations between Structure and Morphology of Crystals. II. Acta. Crystallogr. 1955, 8, 521-524.

(29) Curie, P. Sur la Formation des Cristaux et sur les Constantes Capillaires de Leurs Différentes Faces. Bull. Soc. Fr. Mineral. Cristallogr. 1885, 8, 145-150.

(30) Wulff, G. Zur Frage der Geschwindigkeit des Wachstums und der Auflösung der Kristallflächen. Z. Kristallogr. Mineral. 1901, 34, 449-530. 
(31) Greer, H. F.; Zhou, W. Z. Electron Diffraction and HRTEM Imaging of Beam-Sensitive Materials. Crystallogr. Rev. 2011, 17, $163-185$.

(32) Wright, P. A.; Zhou, W. Z.; Perez-Pariente, J.; Arranz, M. Direct Observation of Growth Defects in Zeolite Beta. J. Am. Chem. Soc. 2005, 127, 494-495.

(33) Hong, S. B.; Lear, E. G.; Wright, P. A.; Zhou, W. Z.; Cox, P. A.; Shin, C. H.; Park, J. H.; Nam, I. S. Synthesis, Structure Solution, Characterization, and Catalytic Properties of TNU-10: A High-Silica Zeolite with the STI Topology. J. Am. Chem. Soc. 2004, 126, 58175826.

(34) Geng, J. F.; Zhou, W. Z.; Skelton, P.; Yue, W. B.; Kinloch, I. A.; Windle, A. H.; Johnson, B. F. G. Crystal Structure and Growth Mechanism of Unusually Long Fullerene $\left(\mathrm{C}_{60}\right)$ Nanowires. J. Am. Chem. Soc. 2008, 130, 2527-2534.

(35) Xiao, B.; Byrne, P. J.; Wheatley, P. S.; Wragg, D. S.; Zhao, X. B.; Fletcher, A. J.; Thomas, M.; Peters, L.; Evans, J. S. O.; Warren, J. E.; Zhou, W. Z.; Morris, R. E. Chemically Blockable Transformation and Ultraselective Low-Pressure Gas Adsorption in a Non-Porous Metal Organic Framework. Nat. Chem. 2009, 1, 289-294.

(36) Greer, H. F.; Wheatley, P. S.; Ashbrook, S. E.; Morris, R. E.; Zhou, W. Z. Early Stage Reversed Crystal Growth of Zeolite A and Its Phase Transformation to Sodalite. J. Am. Chem. Soc., 2009, 131, 17986-17992.

(37) Yao, J. F.; Li, D.; Zhang, X. Y.; Kong, C. H.; Yue, W. B.; Zhou, W. Z.; Wang, H. T. Cubes of Zeolite A with an Amorphous Core. Angew. Chem. Int. Ed. 2008, 47, 8397-8399.

(38) Self, K.; Zhou, H.; Greer, H. F.; Tian, Z. R.; Zhou, W. Z. Reversed Crystal Growth of $\mathrm{ZnO}$ Microdiscs. Chem. Commun. 2013, 49, 5411-5413.

(39) Richie, A. W.; Watson, M. I. T.; Turnbull, R.; Lu, Z. Z.; Telfer, M.; Gano, J. E.; Self, K.; Greer, H. F.; Zhou, W. Z. Reversed Crystal Growth of Rhombohedral Calcite in the Presence of Chitosan and Gum Arabic. CrystEngComm 2013, 15, 10266-10271.

(40) Rowsell, J. L. C.; Yaghi, O. M. Strategies for Hydrogen Storage in Metal-Organic Frameworks. Angew. Chem. Int. Ed., 2005 , $44,4670-4679$.
(41) Kim, H.; Das, S.; Kim, M. G.; Dybtsev, D. N.; Kim, Y.; Kim, K. Synthesis of Phase-pure Interpenetrated MOF-5 and Its Gas Sorption Properties. Inorg. Chem. 2011, 50, 3691-3696.

(42) Xu, D.; Ma, Y.; Jing, Z.; Han, L.; Singh, B.; Feng, J.; Shen, X.; Cao, F.; Oleynikov, P.; Sun, H.; Teraski, O.; Che, S. $\pi-\pi$ Interaction of Aromatic Groups in Amphiphilic Molecules Directing for SingleCrystalline Mesostructured Zeolite Nanosheets. Nat. Comm. 2014, 5 , 4262.

(43) Zhang, X.; Liu, D.; Xu, D.; Asahina, S.; Cychosz, K. A.; Agrawal, K. V.; Wahedi, Y. A.; Bhan, A.; Hashimi, S. A.; Terasaki, O.; Thommes, M.; Tsapatsis, M. Synthesis of Self-Pillared Zeolite Nanosheets by Repetitive Branching. Science 2012, 336, 1684-1687.

(44) Chaikittisilp, W.; Suzuki, Y.; Mukti, R. R.; Suzuki, T.; Sugita, K.; Itabashi, K.; Shimojima, A.; Okubo, T. Formation of Hierarchically Organized Zeolites by Sequential Intergrowth. Angew. Chem. Int. Ed. 2013, 52, 3355-3359.

(45) Liu, L.; Wang, H.; Wang, R.; Sun, C; Zeng, S.; Jiang, S.; Zhang, D.; Zhu, L.; Zhang, Z. N-Methyl-2-pyrrolidone Assisted Synthesis of Hierarchical ZSM-5 with House-of-Cards-Like Structure. RSC Adv. 2014, 4, 21301-21305.

(46) Greer, H. F. Non-Classical Crystal Growth of Inorganic and Organic Materials. Mater. Sci. Technol. 2014, 30, 611-626.

(47) Feng, Y.; Jiang, H.; Chen. M.; Wang, Y. Construction of an Interpenetrated MOF-5 with High Mesoporosity for Hydrogen Storage at Low Pressure. Powder Technol. 2013, 249, 38-42. 
Synthesis and Formation Mechanism of Textured MOF-5

Heather F. Greer, Yuehao Liu, Alex Greenaway, Paul A. Wright and Wuzong Zhou

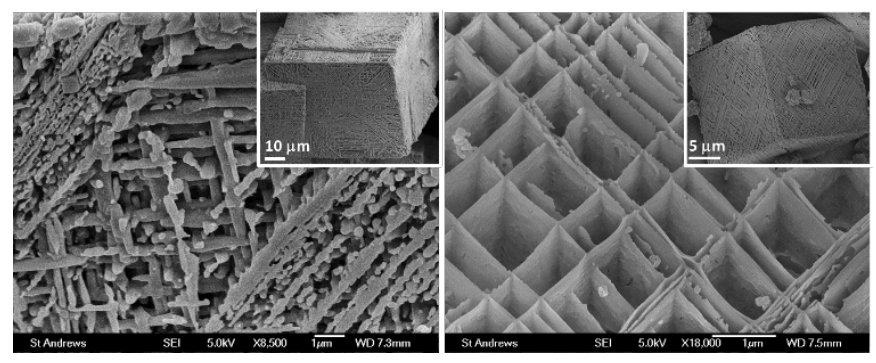

Synopsis: We present a non-classical crystal growth mechanism for cubic MOF-5 crystals with textured and house-of-card surface structures based on the electron microscopic analysis of specimens with different reaction times. Extended reaction times generated MOF- 5 cubes with a single crystal-like appearance despite their hidden three tier structure containing varying porosities hidden underneath. This is evidence of a reversed crystal growth process. 